\title{
Effects of Non-Destructive Overloading on the Mechanical Properties, Microstructure and Ultrastructure of Rabbit Patellar Tendons*
}

\author{
Ei YAMAMOTO** and Kousuke OHTA*** \\ **Department of Mechanical Engineering and Biomimetics, \\ Faculty of Biology-Oriented Science and Technology, \\ Kinki University, Wakayama 649-6493, Japan \\ E-mail: ei@waka.kindai.ac.jp \\ ${ }^{* * *}$ Graduate School of Biology-Oriented Science and Technology, \\ Kinki University, Wakayama 649-6493, Japan
}

\begin{abstract}
Incomplete or subfailure injuries of tendons occur more frequently than their complete ruptures. However, the mechanical behavior of the incompletely injured tissues is poorly understood. In the present study, we quantified the mechanical properties, microstructure, and ultrastructure of rabbit patellar tendons subjected to non-destructive overloading. No significant changes in the mechanical properties were observed in the tendon specimens which were applied a subfailure stretch equivalent to $80 \%$ of the failure stress of the control tendons at a low strain rate $(1.67 \% / \mathrm{sec})$. There was a significant decrease not in the tensile strength but in the tangent modulus of the specimens when they were subjected to $90 \%$ of the control failure stress at the low rate. For the same level of overloading at a high strain rate $(16.7 \% / \mathrm{sec})$, both the modulus and strength of the overloaded specimens were significantly lower than those of the control ones. Microstructually, irrespective of the strain rate, crimped collagen fibers in the overloaded tendons were straightened by the $90 \%$ overloading. In contrast, adverse alterations in the ultrastructure were induced by the $90 \%$ overloading only at the high rate. These results indicate that tendinous tissues have potential capacities to maintain the original strength if a relatively high overload is monotonically applied at lower strain rates. However, it is possible that subfailure overloads delivered at higher strain rates produce more severe changes in the properties and structures of the tissues.
\end{abstract}

Key words: Biomechanics, Patellar Tendon, Subfailure Injury, Overloading, Tensile Property, Strain Rate Effect, Crimp Structure, Collagen Fibril

\section{Introduction}

Motor vehicle accidents and competitive sports frequently cause injuries of such musculoskeletal soft tissues as tendons. Their injuries range in severity from a simple sprain to a complete rupture. Joint sprains are regarded as the incomplete or partial failures of the structure of tendons. These types of injuries have a high incidence among the joint traumas ${ }^{(1,2)}$. In sports, tendinous partial tears are common injuries in shoulder, ankle, and knee joints. Although many patients of such traumas can return to active sports, they may do so at a lower level of activity than before the injuries. Moreover, the partial tears may result in increased joint instability, even after a prolonged period of time. 
Many previous studies have been performed to quantify the biomechanical characteristics of tendons, focusing on their mechanical parameters concerned with the complete ruptures ${ }^{(3-5)}$. In contrast, the effects of non-destructive overloading on the mechanical parameters have not been studied well, due to the complex and various modes of the incomplete failures. Therefore, little information is available to know the basic phenomena of subfailure injuries as well as the failure mechanisms. In particular, rigorous relationships between subfailure injuries and mechanical stress remain unknown. A few quantitative studies on the subfailure injuries of ligaments have been conducted by Panjabi and his colleague ${ }^{(6-9)}$. In their studies, ligamentous tissues were subjected to a subfailure stretch which was equivalent to $80 \%$ of the failure deformation of the intact ligaments, and they investigated the shape of load-deformation curve, failure load, and failure deformation of the overloaded tissues. That is, they focused on the changes in the structural properties due to overloading. There have been no reports on the effects of subfailure overloading on the changes in the material properties such as failure stress and strain. In addition, no quantitative attempts have been conducted to investigate the microstructural and ultrastructural alterations of tendons subsequent to well defined subfailure stretches.

Based on the assumption that the biomechanical behavior after overloading enables us to elucidate the weakening of damaged tissues, our overall goal was to quantify structurefunction relationships in incompletely injured tendinous tissues. For this goal, we investigated the mechanical properties, microstructure, and ultrastructure of rabbit patellar tendons after they were overloaded monotonically. Specific objectives in the present study were to: (1) quantify the stress magnitude of overloading that induces detrimental changes in the biomechanical properties, (2) investigate the strain rate dependence of sub failure injuries, and (3) know the differences in fibrillar structures at microscopic and nanoscopic levels between the normal and overloaded tissues. Findings will provide an insight into the failure mechanisms of incompletely injured tendons. Moreover, a more complete characterization of the strength reduction that occurs after non-destructive overloading may be implicated in the improvement of diagnostic techniques and rehabilitation programs for tendinous injuries.

\section{Materials and Methods}

Animal experimentation in the present study was carried out under the guideline of the Animal Care and Use Committee of Faculty of Biology-Oriented Science and Technology, Kinki University.

\subsection{Specimen Preparation}

Twenty-seven skeletally matured female Japanese white rabbits $(3.2 \pm 0.2 \mathrm{~kg})$ were obtained after sacrifice from other research laboratories at Faculty of Biology-Oriented Science and Technology, Kinki University. None of the rabbits used for the present experiment had been involved in prior research that would have subjected them to any musculoskeletal damage. Twenty-one rabbits of them were used for biomechanical tests. Remaining ones were used for microstructural and ultrastructural observations.

The patellar tendon with the entire patella and tibia (patellar tendon-bone complex) was harvested from both hindlimbs of each animal. The right and left tendons were grouped as a pair, wrapped in gauze that had been soaked in a physiological saline solution, and stored at $-20^{\circ} \mathrm{C}$ until required for testing. After thawing, the medial and lateral one-thirds of all of the paired tendons were dissected along the longitudinal axis using a surgical knife, and the remaining central one-third was used for following biomechanical tests. Previous studies have shown that failure always occurred in the patella, if we used the patella-whole patellar tendon-tibia complex for tensile testing ${ }^{(4,10)}$. This happened because the midsubstance of 
the patellar tendon was stronger than the patella. To determine the effects of overloading on the tendon substance, in the present study, we used only the central one-third tendon for biomechanical testing ${ }^{(4,10)}$. Non-destructive monotonic overloads were applied to left patellar tendons, whereas right patellar tendons were used to obtain the mechanical properties of normal tissues for the determination of the stress magnitude in overloading procedures. In order to obtain the control data on structures, other two normal right patellar tendons were used for microstructural and ultrastructural observations.

\subsection{Measurement of Cross-Sectional Area}

Prior to tensile testing, the cross-sectional area of each specimen was measured with a non-contact optical technique. The area measurement was conducted using a specially designed apparatus (Fig. 1 (a)) which comprises two laser displacement meters (LK-G80, Keyence, Osaka, Japan). A constant tensile load of $0.5 \mathrm{~N}$ was applied to the specimen. When it was placed between two laser beams, its thickness at a position was measured with the resolution of $1 \mu \mathrm{m}$. The data were recorded in a personal computer. Such thickness measurements were made at intervals of $100 \mu \mathrm{m}$ throughout whole width of the specimen. From these thickness data, the cross-sectional shape was reconstructed. Then, the crosssectional area was calculated with a trapezoidal rule. The area measurements were done at the middle of each specimen, at $2 \mathrm{~mm}$ proximal position from the middle, and at $2 \mathrm{~mm}$ distal position to the middle. An averaged cross-sectional area was obtained from these three sets of measurements.

\subsection{Mechanical Loading Procedures}

The patella and the tibia were cast separately in small aluminum pots with use of polymethylmethacrylate resin. Two parallel lines were drawn on the surface of a patellar tendon approximately $15 \mathrm{~mm}$ apart using a stain (Nigrosine), which were used for the measurement of strain with a video dimension analyzer (C3160, Hamamatsu Photonics, Hamamatsu, Japan) (Fig. 1 (b)). The tendinous substance was kept wet with a physiological saline solution during preparation and test arrangement. In the similar fashion to that reported previously ${ }^{(4)}$, the bone-tendon specimen was mounted onto a set of specially designed grips using the aluminum pots, and attached to a conventional tensile tester (AGSH5kN, Shimadzu, Kyoto, Japan), with the tibia being inclined at 45 degrees against the patella (Fig. 1 (b)). The specimen was immersed in a physiological saline solution of $37^{\circ} \mathrm{C}$ during mechanical testing.

In our preliminary experiment, we examined the differences in the mechanical properties between the right and left patellar tendons. The tangent modulus, tensile strength, and strain at failure of the right tendons were $954 \pm 640 \mathrm{MPa}(\mathrm{n}=5$, Mean \pm S.D.), $50.8 \pm$ $7.4 \mathrm{MPa}$, and $7.8 \pm 2.4 \%$, respectively. Those parameters in the left tendons were $889 \pm 307$ $\mathrm{MPa}, 48.9 \pm 12.3 \mathrm{MPa}$, and $7.3 \pm 3.4 \%$, respectively. Paired t-tests showed that there were no significant differences in the tangent modulus $(p=0.84)$, tensile strength $(p=0.78)$, and strain at failure $(p=0.78)$ between the right and left tendons. Based on such preliminary results, the right and left tendons were used for the Control and Overload groups, respectively. Twenty-one right patellar tendons (Control group) of the pair were stretched until failure to obtain the biomechanical properties of normal, contralateral tissues. The specimen was applied a preload of $0.5 \mathrm{~N}$, followed by ten cycles of loading and unloading between the displacement of 0 and $0.5 \mathrm{~mm}$ at a strain rate of $1.67 \% / \mathrm{sec}$. The initial length of the midsubstance and the crosshead speed of the tester were approximately $20 \mathrm{~mm}$ and $20 \mathrm{~mm} / \mathrm{min}$, respectively. Subsequently to this preconditioning, each control specimen was stretched to failure at the same rate. Twenty-one left patellar tendons (Overload group) were divided into three experimental groups depending on the magnitude $(80 \%$ or $90 \%$ of the failure stress of the contralateral tendon) and the strain rate $(1.67$ or $16.7 \% / \mathrm{sec})$ of 
overloading: Ov80-1.67\%/s, Ov90-1.67\%/s, and Ov90-16.7\%/s groups for 7 specimens for each. The same preconditioning was conducted for the specimen in the Overload group. At the strain rate of $1.67 \% / \mathrm{sec}$, it was then applied a single subfailure stretch that was $80 \%$ (Ov80-1.67\%/s group) or 90\% (Ov90-1.67\%/s group) of the failure stress of the contralateral tendon. In addition, we applied an overload of $90 \%$ of the failure stress to the specimen at the strain rate of $16.7 \% / \mathrm{sec}$ (Ov90-16.7\%/s group) in order to investigate the strain rate dependence of subfailure injuries. Following the non-destructive overloading, each overloaded specimen was stretched to failure to know the changes in the mechanical properties. A previous study ${ }^{(11)}$ on the in vivo tension applied to the rabbit patellar tendon showed that the strain rate of the tension is not far from $1.67 \% / \mathrm{sec}$ during normal walking. From tensile testing, we obtained a stress-strain curve, tangent modulus, tensile strength, and strain at failure of each specimen. The tangent modulus was defined as the slope of each stress-strain curve between $1 \%$ and $3 \%$ with a least-squares method.

For microstructural and ultrastructural studies, we used other 4 specimens which were applied the $90 \%$ overload at $1.67 \% / \mathrm{sec}$ (Ov90-1.67\%/s group) or $16.7 \% / \mathrm{sec}$ (Ov90$16.7 \%$ /s group). As shown in the Results section, the changes in the mechanical properties were not statistically significant in the case of the $80 \%$ overload at $1.67 \% / \mathrm{sec}$. Therefore, no microstructural and ultrastructural observations were conducted in the Ov80-1.67\%/s group.

(a)

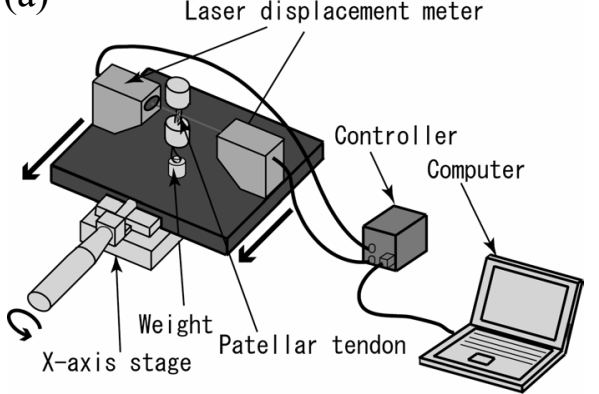

(b)

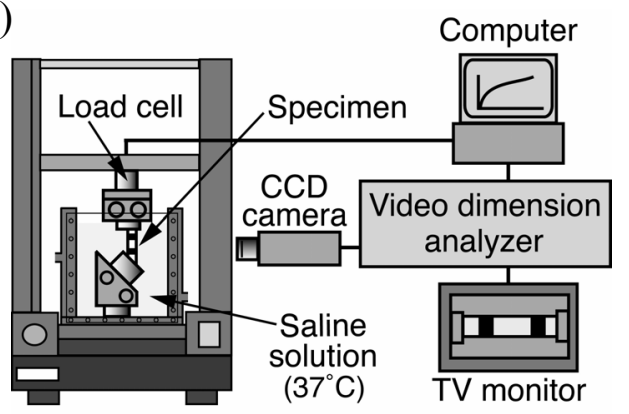

Fig. 1 Apparatus for the measurement of cross-sectional area of patellar tendons (a) and a tensile tester used to determine the mechanical properties of the tendons (b).

\subsection{Microstructural Observation}

The specimens in the Ov90-1.67\%/s and Ov90-16.7\%/s groups were used to observe the crimp morphology of collagen fibers. While immersing in a physiological saline solution of room temperature under no load condition, we photographed the specimen through a phase contrast light microscope (IX71, Olympus, Tokyo, Japan). From the picture, crimp angle $(\theta)$ and crimp length $\left(\mathrm{d}_{0}\right)$ were measured with a digital caliper and a graduator having the accuracies of $10 \mu \mathrm{m}$ and $0.1^{\circ}$, respectively. We obtained these parameters from 6 locations randomly selected in each specimen. Definition of each parameter expressing the crimp morphology is shown in Fig. $2^{(12,13)}$. First, a straight line was drawn between a top point of the waveform of the fibril and the neighboring two bottom points, and the distance between the top and bottom points (crimp length, $\mathrm{d}_{0}$ ) was measured. The midpoint of the line connecting the top and bottom points was called middle point. Crimp angle $\left(\theta_{0}\right)$ was defined as the angle between the straight line and the line connecting neighboring two middle points. Similar observation and analysis of the crimp morphology were conducted for non-treated, intact specimens (Control group). 


\subsection{Ultrastructural Observation}

After the overloading procedures, tendon segments were prepared for routine transmission electron microscopy. Each specimen was transversely divided into six blocks. From each block, $1 \mathrm{~mm}$-thick segments sliced perpendicular to the patellar tendon axis were dissected using a surgical blade, fixed in a $2.5 \%$ buffered glutaraldehyde solution, and postfixed in $1 \%$ osmium tetroxide buffer solution. The segment was dehydrated in graded alcohols and embedded in epoxy resin. Ultrathin transverse sections of approximately 80 $\mathrm{nm}$ in thickness were cut out from each segment. The section was stained with uranyl acetate and lead citrate for ultrastructural observations using a transmission electron microscopy (H-7100TE, Hitachi, Tokyo, Japan). Electron micrographs of the three experimental groups (Ov90-1.67\%/s, Ov90-16.7\%/s, and Control groups) were taken at a magnification of 20,000 . The fibril occupation ratio $\left(C_{F}\right)$ and the number of collagen fibrils in $1 \mu^{2} \mathrm{~m}^{2}\left(\mathrm{~N}_{\mathrm{F}}\right)$ were determined from these micrographs with use of image software, where the fibril occupation ratio was defined as the ratio of the total cross-sectional area of collagen fibrils to the whole visualized area.

\subsection{Statistical Analysis}

All data were expressed as mean \pm S.D. The differences in the mechanical parameters between the Ov80-1.67\%/s and Control groups were analyzed by a paired t-test with a significance level of 0.05 . It was also used to compare the mechanical properties between the Control and Overload groups in case of the specimens subjected to $90 \%$ of the normal failure stress with two levels of strain rate (Ov90-1.67\%/s and Ov90-16.7\%/s groups).

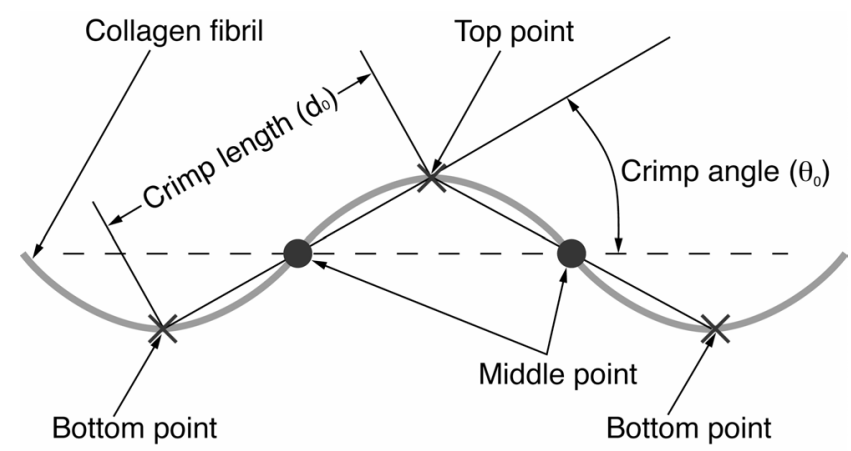

Fig. 2 Definition of crimp parameters of collagen fibrils in the patellar tendon ${ }^{(12,13)}$.

\section{Results}

\subsection{Change in the Mechanical Properties}

The stress-strain curve in the Ov80-1.67\%/s group is shown in Fig. 3 (a), together with that in the Control group. Each curve was almost linear between $1 \%$ and $3 \%$ strains, with a toe region under $1 \%$ strain. There were no statistically significant differences in the tangent modulus $(\mathrm{p}=0.418)$, tensile strength $(\mathrm{p}=0.604)$, and strain at failure $(\mathrm{p}=0.527)$ between the Ov80-1.67\%/s and Control groups (Fig. 3 (b)). In the case of the specimens applied an overload that was $90 \%$ of the normal failure stress with two levels of strain rate, there were no noticeable differences in the shape of the stress-strain curves among the Ov $90-1.67 \% / \mathrm{s}$, Ov90-16.7\%/s, and Control groups (Fig. 4 (a)). Figure 4 (b) shows the mechanical properties in these three groups. As compared with the Control group, significant reductions in the tangent modulus were observed in the Ov90-1.67\%/s $(\mathrm{p}=0.014)$ and Ov90-16.7\%/s $(\mathrm{p}=0.002)$ groups. The modulus in the Ov90-1.67\%/s and Ov90-16.7\%/s groups was approximately $57 \%$ and $44 \%$ of the control value, respectively. While there was no 
significant difference in the tensile strength between the Ov90-1.67\%/s and Control groups $(\mathrm{p}=0.914)$, the strength in the Ov90-16.7\%/s group was significantly lower than that in the Control group $(p=0.008)$. The strength in the Ov90-16.7\%/s group was approximately $67 \%$ of the control value. No significant differences in the strain at failure were observed between the Control and Overload groups.

(a)

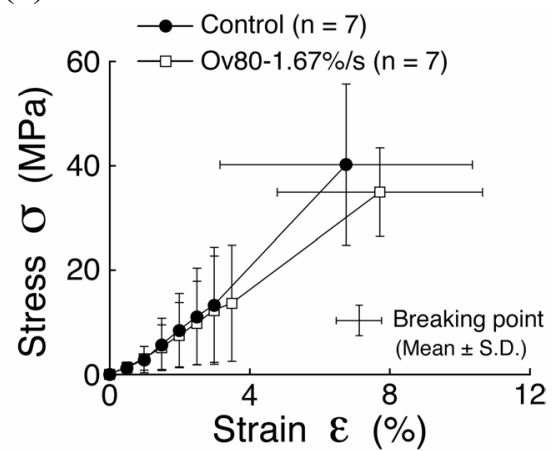

(b) $\square$ Control $(n=7)$

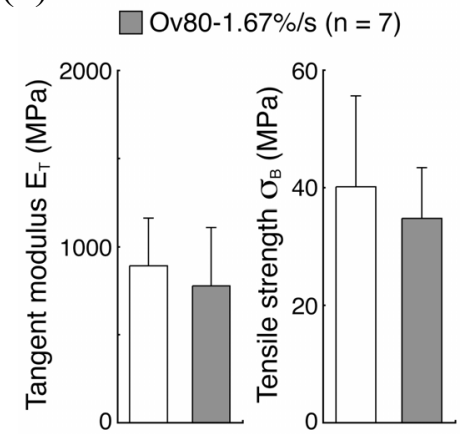

Fig. 3 Stress-strain curves (a) and tensile properties (b) of the control specimens and the specimens overloaded to $80 \%$ of the failure stress of the normal tendons at the strain rate of $1.67 \% / \mathrm{sec}$ (Ov80-1.67\%/s group).

(a)

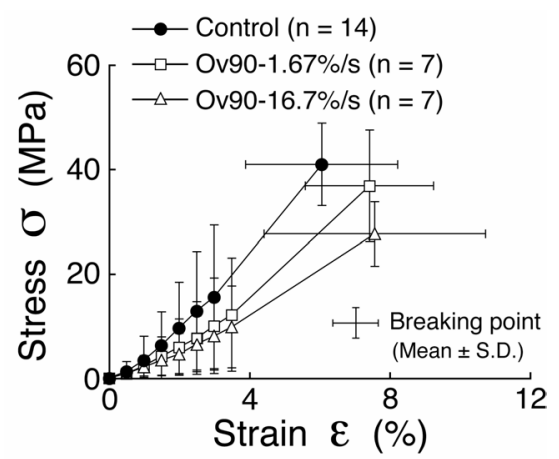

(b)

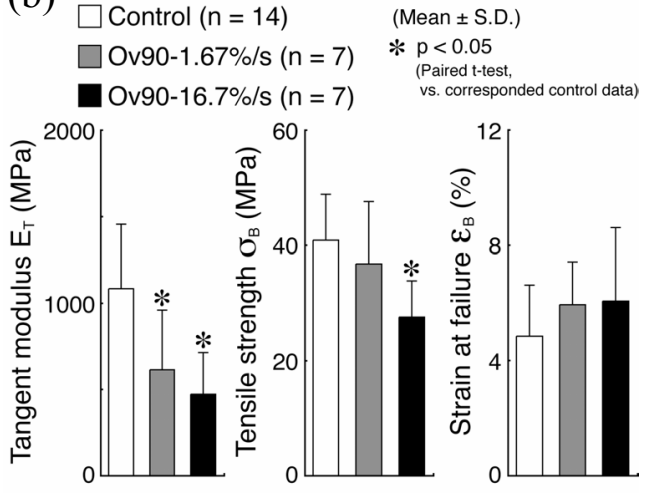

Fig. 4 Stress-strain curves (a) and tensile properties (b) of the control specimens and the specimens overloaded to $90 \%$ of the failure stress of the normal tendons at the strain rate of 1.67 (Ov90-1.67\%/s group) and 16.7\%/sec (Ov90-16.7\%/s group).

\subsection{Change in the Microstructure}

Figure 5 (a) shows examples of optical micrographs displaying the crimp morphology in the control and overloaded specimens. The averaged crimp angle $\left(\theta_{0}\right)$ was approximately $35^{\circ}, 19^{\circ}$, and $12^{\circ}$ in the Control, Ov90-1.67\%/s, and Ov90-16.7\%/s groups (Fig. 5 (b)). Remarkable reductions were observed in the crimp angle of the overloaded specimens, indicative of the straightening of the crimp of collagen fibers. The angle appeared to decrease with increase in the strain rate of overloading. That in the Ov90-1.67\%/s and Ov $90-16.7 \%$ /s groups was $54 \%$ and $34 \%$ of the control value, respectively. Furthermore, the crimp length $\left(\mathrm{d}_{0}\right)$ of the overloaded specimens (approximately 45 and $48 \mu \mathrm{m}$ in the Ov90$1.67 \% / \mathrm{s}$ and Ov90-16.7\%/s groups, respectively) was obviously different from that of the control one (approximately $34 \mu \mathrm{m}$ ) (Fig. 5 (b)). The length appeared to increase with increase in the strain rate of overloading. That in the Ov90-1.67\%/s and Ov90-16.7\%/s groups was approximately $133 \%$ and $142 \%$ of the control value, respectively. 


\subsection{Change in the Ultrastructure}

Figure 6 (a) shows examples of electron micrographs $(2.2 \times 1.6 \mu \mathrm{m}$ in image size $)$ displaying collagen fibrils in the control and overloaded specimens. The fibril diameters in the control specimen ranged from 30 to $350 \mathrm{~nm}$. Ultrastructural changes in the Ov90$16.7 \% / \mathrm{s}$ group were more remarkable than those in the Ov90-1.67\%/s group (Fig. 6 (b)). The fibril occupation ratio $\left(\mathrm{C}_{\mathrm{F}}\right)$ was approximately $70 \%$ and $50 \%$ in the Ov90-1.67\%/s and Ov90-16.7\%/s groups, respectively, whereas the ratio was approximately $73 \%$ in the control group. The number of collagen fibrils in $1 \mu \mathrm{m}^{2}\left(\mathrm{~N}_{\mathrm{F}}\right)$ in the Control, Ov90-1.67\%/s and Ov $90-16.7 \% / \mathrm{s}$ groups was $30.2,28.9$, and 26.5 , respectively. Figure 7 shows the distribution of fibril diameters in total visualized area, which was obtained from 6 images in each group. In the present study, we divided the fibrils into two groups according to the diameters: those blow $160 \mathrm{~nm}$ and above $160 \mathrm{~nm}$. The value of $160 \mathrm{~nm}$ in diameter was the middle of the diameter range in the control specimen. Irrespective of the strain rate, the number of fibrils with smaller diameters in both the overload groups (Ov90-1.67\%/s and Ov $90-16.7 \% / \mathrm{s}$ groups) was smaller than that in the control specimen. In contrast, a decrease of fibrils with larger diameters was observed only in the Ov90-16.7\%/s group.

(a)
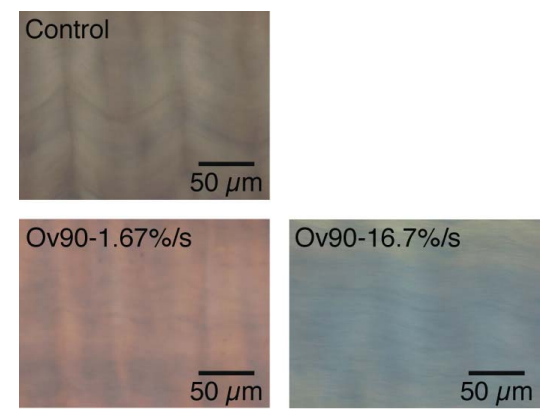

(b)

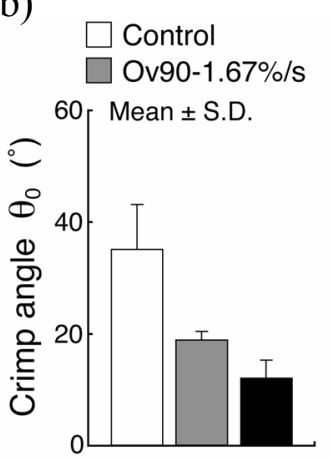

Ov90-16.7\%/s

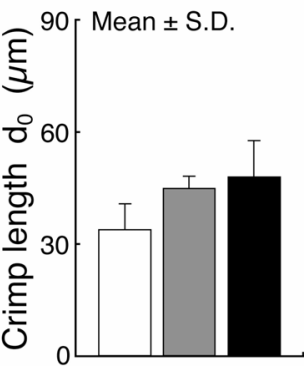

Fig. 5 Optical micrographs of crimp morphology (a) and the parameters expressing the morphology (b) of the control and overloaded specimens.

(a)

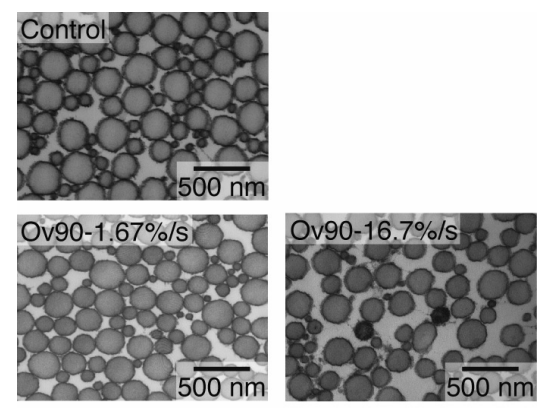

(b)

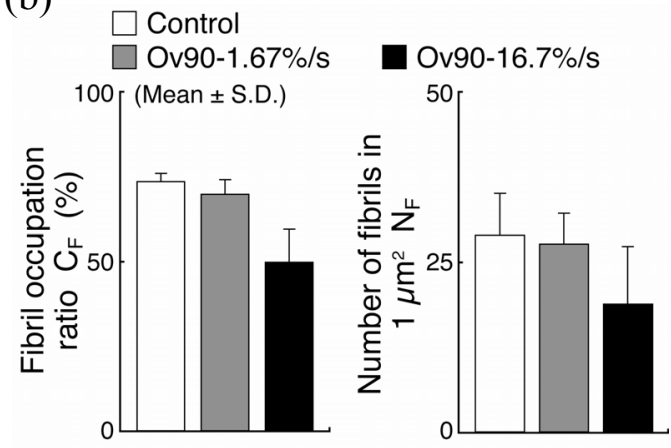

Fig. 6 Electron micrographs of collagen fibrils (a) and the parameters expressing the fibrillar structure (b) of the control and overloaded specimens. 


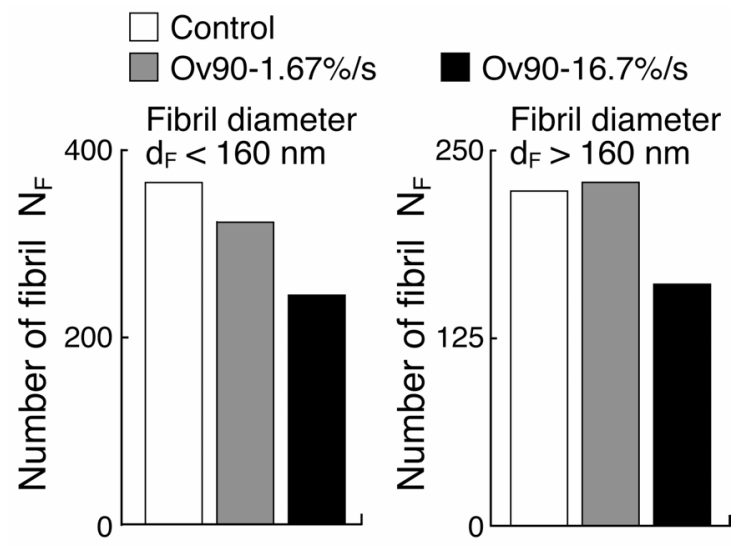

Fig. 7 Number of collagen fibrils having a diameter of above or blow $160 \mathrm{~nm}$ in total visualized area of the control and overloaded specimens.

\section{Discussion}

Although incomplete failures of tendons occur more frequently than their complete ruptures, the mechanical behavior of the tissues after non-destructive overloading have not been studied well. Only a few biomechanical studies on the subfailure injuries of ligaments have been conducted by Panjabi and his colleague ${ }^{(6-9)}$. In their studies, ligamentous tissues were subjected to a subfailure stretch which was equivalent to $80 \%$ of the failure deformation of the intact ligament. After such subfailure deformation, they examined the changes in the shape of load-deformation curve as well as the failure load and failure deformation. That is, the previous studies on the subfailure injuries have been based on the knowledge of the structural characteristics of ligamentous tissues, including the effects of their size and shape. To date, there have been no quantitative studies on the effects of subfailure overloading, focusing on the changes in the material properties such as failure stress and failure strain. In addition, no attempts have been conducted to investigate the effects of the stress magnitude and strain rate of overloading on the material properties. In the present study, the specimens prepared from rabbit patellar tendons were monotonically overloaded to $80 \%$ and $90 \%$ of the failure stress of the normal, control tissues. It has been suggested that in vivo tensions applied to tendinous tissues are less than $30 \%$ of their failure stress ${ }^{(4,14)}$. Therefore, our overload levels were outside the physiological range of in vivo stresses applied to tendinous tissues. After such high levels of overloading, we determined the mechanical properties, microstructure, and ultrastructure of the incompletely injured tendons.

Although there were no significant reductions in the tangent modulus and tensile strength in the Ov80-1.67\%/s group, a significant reduction in the tangent modulus was observed in the Ov90-1.67\%/s group. These results suggest that there is a threshold level of the stress magnitude for tendinous subfailure between $80 \%$ and $90 \%$ of the failure stress of the normal tissues. However, no significant reduction in the tensile strength was observed in the Ov90-1.67\%/s group, indicative of less sensitivity of the tensile strength to nondestructive overloading than the tangent modulus. Essentially similar alternations were observed in a previous study ${ }^{(6)}$ on the structural properties of rabbit anterior cruciate ligaments applied a subfailure stretch that was $80 \%$ of the normal failure deformation. The previous finding showed that none of the failure parameters such as failure load and failure deformation were significantly altered following the subfailure injury, although the changes due to the injury were mostly seen in the shape of the load-deformation curve at low load levels. From these results, we can say that tendons and ligaments have potential capacities to keep the original tensile strength if a relatively high overload is applied monotonically. A 
few studies on the fatigue behavior of tendons ${ }^{(15,16)}$ demonstrated that the complete failure occurs under cyclic loading at a low magnitude of load, which monotonic itself will not result in the subfailure injuries. Taken together, it is suggested that monotonic overloading produced less serious strength reductions than prolonged cyclic loading. These findings also possibly support the concept that overuse failures in tendons are prevented by an early cessation of loading. However, our data showed that monotonic overloading at a low strain rate induced a significant decrease in the tangent modulus, which may alter joint kinematics and activity levels of the patient with subfailure tendinous injuries.

With regards to the strain rate effects, our results showed that the tangent modulus and tensile strength of the overloaded specimens at the high strain rate decreased more remarkably compared to those at the low rate. Many previous studies have been done on the effects of strain rate on the failure properties of tendons and ligaments ${ }^{(17-20)}$. By contrast, no studies have addressed the strain rate dependence of the subfailure injuries, except for the work done by Panjabi and Courtnet ${ }^{(9)}$ on the rabbit anterior cruciate ligament. They reported that the failure deformation of the ligament applied the high-speed overloading (1 $\mathrm{m} / \mathrm{s})$ increased more markedly than that applied the slow-speed one $(0.001 \mathrm{~m} / \mathrm{s})$. This result is essentially similar to that obtained from the present study. That is, the subfailure overloading delivered at higher rates produces more severe changes in the mechanical properties of tendons and ligaments.

There have been a few biomechanical studies on the subfailure injuries of and ligaments ${ }^{(6-9)}$. However, the present study is the first attempt to quantitatively determine the effects of non-destructive overloading on the microstructural and ultrastructural characteristics of tendinous tissues. With regard to the crimp morphology, collagen fibers in the tissues were straightened by the application of overloading. Such remarkable changes induced by non-destructive overloading were observed both in the Ov90-1.67\%/s and Ov90-16.7\%/s groups. This result implies that irreversible, residual strains are developed in the overloaded tendons after subfailure injuries such as sprains or dislocations. These residual deformations of tendinous tissues may be clinically implicated in prolonged joint loosening and instability. Ultrastructurally, the collagen fibril structure was markedly altered only in the Ov90-16.7\%/s group, while there were no notable differences in the ultrastructure between the Control and Ov90-1.67\%/s groups. This result demonstrated that ultrastructural damages under overloading at the high strain rate were more severe than under that at the low rate. Therefore, it is likely that the strain rate dependence of subfailure injuries at the microstructural level is different from that at the ultrastructural level. Furthermore, the number of fibrils with small diameters decreased both in the Ov90$1.67 \% / \mathrm{s}$ and Ov90-16.7\%/s groups, whereas that with large diameters decreased only in the Ov90-16.7\%/s group. These ultrastructural results appear to correspond with biomechanical results. That is, it is possible that the decrease in the tangent modulus and the tensile strength is attributable to the damage of fibrils with small and large diameters, respectively. The microstructural and ultrastructural findings obtained from the present study are presumably important for understanding the failure mechanisms of tendons under nondestructive overloading.

The present study is novel in a number of ways: its application of overloading based on the stress criteria, its determination of the material properties after overloading, and its contrast of the effects of overloading at high versus low strain rate on the mechanical properties and structures. Despite these novelties, some limitations should be considered. First, the non-destructive overloads were applied to the tendon specimens at relatively low strain rates $(1.67$ and $16.7 \% / \mathrm{sec})$. Tendons are frequently injured by sports activities and automobile accidents at much higher strain rates than those in laboratory tests. The strain rates of more than $50 \% / \mathrm{sec}$ were considered to be reasonable estimates of the rates in these injuries ${ }^{(21)}$. Second, we made no statistical comparisons in the parameters expressing the 
crimp morphology and fibrillar structure among the groups, because only one specimen of each group was used for each observation. And third, we examined the mechanical properties of devitalized tendons immediately after the in vitro application of overloading. The contribution of biological response to the non-destructive overloading is likely to be related the etiology of the chronic instabilities of joints after the subfailure injuries. Further biomechanical, biochemical, and morphological studies should be conducted to elucidate the mechanisms of tendinous microdamages in the incompletely injured tissues.

In summary, the subfailure injuries of tendons depended upon not only the magnitude but also the strain rate of non-destructive overloading. At the low strain rate, the mechanical properties were not significantly altered by an overload equal to $80 \%$ of the failure stress of the normal tissues, while a significant decease was observed in the tangent modulus following an overload that was $90 \%$ of the failure stress. Moreover, both the tangent modulus and tensile strength were significantly reduced by the $90 \%$ overloading at the high rate. Adverse changes in the ultrastructure due to the $90 \%$ overloading were observed only in the case of the high rate. These results indicate that tendinous tissues have potential capacities to maintain the original strength if a relatively high overload is applied monotonically. However, it is possible that subfailure injuries delivered at high strain rates produce more detrimental changes in the properties and structures of tendons. We conclude from our findings that non-destructive overloading has adverse effects on the biomechanical performance of tendons, which may result in increased joint laxity under dynamic in vivo loading conditions.

\section{Acknowledgements}

The authors would like to thank Yoshitaka Horiuchi, Life Science Research Institute, Kinki University, for his technical assistant for ultrastructural observations of tendinous tissues using a transmission electron microscopy. This study was financially supported in part by the Special-Grant-in-Aid for Scientific Research (07-IV-4) from Faculty of BiologyOriented Science and Technology, Kinki University, and the Matching Fund Subsidy for Private Universities from the Ministry of Education, Culture, Sports, Science and Technology, Japan.

\section{References}

(1) DeHaven, K. E., and Lintner, D. M., Athletic Injuries: Comparison by Age, Sport, and Gender, American Journal of Sports Medicine, Vol. 14, No. 3 (1986), pp. 218-224.

(2) Adirim, T. A., and Cheng, T. L., Overview of Injuries in the Young Athlete, Sports Medicine, Vol. 33, No. 1 (2003), pp. 75-81.

(3) Butler, D. L., Kay, M. D., and Stouffer, D. C., Comparison of Material Properties in Fascicle-Bone Units from Human Patellar Tendon and Knee Ligaments, Journal of Biomechanics, Vol. 19, No. 6 (1986), pp. 425-432.

(4) Yamamoto, N., Hayashi, K., Ohno, K., Yasuda, K., and Kaneda, K., Mechanical Properties of the Rabbit Patellar Tendon, Transactions of the American Society of Mechanical Engineers, Journal of Biomechanical Engineering, Vol. 114, No. 3 (1992), pp. 332-337.

(5) Wren, T. A., Yerby, S. A., Beaupré, G. S., and Carter, D. R., Mechanical Properties of the Human Achilles Tendon, Clinical Biomechanics, Vol. 16, No. 3 (2001), pp. 245-251.

(6) Panjabi, M. M., Yoldas, E., Oxland, T. R., and Crisco, J. J., 1996. Subfailure Injury of the Rabbit Anterior Cruciate Ligament, Journal of Orthopaedic Research, Vol. 14, No. 2 (1996), pp. 216-222. 
(7) Panjabi, M. M., Moy, P., Oxland, T. R., and Cholewicki, J., Subfailure Injury Affects the Relaxation Behavior of Rabbit ACL, Clinical Biomechanics, Vol. 14, No. 1 (1999), pp. 2431.

(8) Panjabi, M. M., Huang, R. C., and Cholewicki, J., Equivalence of Single and Incremental Subfailure Stretches of Rabbit Anterior Cruciate Ligament, Journal of Orthopaedic Research, Vol. 18, No. 5 (2000), pp. 841-848.

(9) Panjabi, M. M., and Courtney, T. W., High-Speed Subfailure Stretch of Rabbit Anterior Cruciate Ligament: Changes in Elastic, Failure and Viscoelastic Characteristics, Clinical Biomechanics, Vol. 16, No. 4 (2001), pp. 334-340.

(10) Yamamoto, E., Hayashi, K., and Yamamoto, N., Mechanical Properties of Collagen Fascicles from the Rabbit Patellar Tendon, Transactions of the American Society of Mechanical Engineers, Journal of Biomechanical Engineering, Vol. 121, No. 1 (1999), pp. 124-131.

(11) Yamamoto, N., Hayashi, K., and Hayashi, F., 1992, In Vivo Measurement of Tension in the Rabbit Patellar Tendon, Transactions of Japanese Society of Mechanical Engineers, Series A, Vol. 58, No. 551 (1992), pp. 1142-1147.

(12) Yamamoto, E., Tokura, S., and Hayashi, K., Effects of Cyclic Stress on the Mechanical Properties of Cultured Collagen Fascicles from the Rabbit Patellar Tendon, Transactions of the American Society of Mechanical Engineers, Journal of Biomechanical Engineering, Vol. 125, No. 6 (2003), pp. 893-901.

(13) Yamamoto, E., Kogawa, D., Tokura, S., and Hayashi, K., Biomechanical Response of Collagen Fascicles to Restressing After Stress Deprivation During Culture, Journal of Biomechanics, Vol. 40, No. 9 (2007), pp. 2063-2070.

(14) Juncosa, N., West, J. R., Galloway, M. T., Boivin, G. P., and Butler, D. L., In Vivo Forces Used to Develop Design Parameters for Tissue Engineered Implants for Rabbit Patellar Tendon Repair, Journal of Biomechanics, Vol. 36, No. 4 (2003), pp. 483-488.

(15) Wang, X. T., Ker, R. F., and Alexander, R. M., Fatigue Rupture of Wallaby Tail Tendons, Journal of Experimental Biology, Vol. 198, No. 3 (1995), pp. 847-852.

(16) Schechtman, H., and Bader, D. L., In Vitro Fatigue of Human Tendons, Journal of Biomechanics, Vol. 30, No. 8 (1997), pp. 829-835.

(17) Danto, M. I., and Woo, S. L.-Y., The Mechanical Properties of Skeletally Mature Rabbit Anterior Cruciate Ligament and Patellar Tendon Over a Range of Strain Rates, Journal of Orthopaedic Research, Vol. 11, No. 1 (1993), pp. 58-67.

(18) Lydon, C., Crisco, J. J., Panjabi, M. M., and Galloway, M., Effect of Elongation Rate on the Failure Properties of the Rabbit Anterior Cruciate Ligament, Clinical Biomechanics, Vol. 10, No. 8 (1995), pp. 428-433.

(19) Yamamoto, N., and Hayashi, K., Mechanical Properties of Rabbit Patellar Tendon at High Strain Rate, Bio-Medical Materials and Engineering, Vol. 8, No. 2 (1998), pp. 83-90.

(20) Pioletti, D. P., Rakotomanana, L. R., and Leyvraz, P. F., Strain Rate Effect on the Mechanical Behavior of the Anterior Cruciate Ligament-Bone Complex, Medical Engineering \& Physics, Vol. 21, No. 2 (1999), pp. 95-100.

(21) Crowninshield, R. D., and Pope, M. H., The Strength and Failure Characteristics of Rat Medial Collateral Ligaments, Journal of Trauma, Vol. 16, No. 2 (1976), pp. 99-105. 